\title{
Synthesis and Antibacterial Characterization of Sustainable Nanosilver Using Naturally-Derived Macromolecules
}

\author{
Francis J. Osonga, Victor Kariuki, Idris Yazgan, Apryl Jimenez, David Luther, Jürgen Schulte \& \\ Omowunmi A. Sadik* \\ Center for Advanced Sensors \& Environmental Systems (CASE) \\ Department of Chemistry \\ State University of New York at Binghamton \\ P.O Box 6000
}

Binghamton, NY, 13902

(C) 2015. This manuscript version is made available under the Elsevier user license

http://www.elsevier.com/open-access/userlicense/1.0/ 


\begin{abstract}
Greener nanosynthesis utilizes fewer amounts of materials, water, and energy; while reducing or replacing the need for organic solvents. A novel approach is presented using naturally-derived flavonoids including Quercetin pentaphosphate (QPP), Quercetin sulfonic acid (QSA) and Apigenin Triphosphate (ATRP). These water soluble, phosphorylated flavonoids were utilized both as reducing agent and stabilizer. The synthesis was achieved at room temperature using water as a solvent and it requires no capping agents. The efficiency of the resulting silver nanoparticle synthesis was compared with naturally-occurring flavonoid such as Quercetin (QCR). Results show that QCR reduced $\mathrm{Ag}^{+}$faster followed by QPP, QSA and ATRP respectively. This is the first evidence of direct utilization of QCR for synthesis of silver nanoparticles (AgNPs) in water. The percentage conversion of $\mathrm{Ag}^{+}$to $\mathrm{Ag}^{0}$ was determined to be 96\% after 35 minutes. The synthesized nanoparticles were characterized using Transmission electron microscopy (TEM), Energy dispersive absorption spectroscopy (EDS), UV-vis spectroscopy, High resolution TEM (HR-TEM) with selected area electron diffraction (SAED). The particle sizes ranged from 2-80 nm with an average size of $22 \mathrm{~nm}$ and in the case of ATRP, the nanoparticle shapes varied from spherical to hexagonal with dispersed particle size ranging from 2-30 nm. Crystallinity was confirmed by XRD and the SAED of (111), (200), and the fringes observed in HRTEM images. Results were in agreement with the UV resonance peaks of 369-440 nm. The particles also exhibit excellent antibacterial activity against $S$. epidermidis, $E$. coli and $C$. freundii in water.
\end{abstract}

KEYWORDS: Flavonoids, green synthesis, nanoparticles, antibacterial, total environment 
The concept of sustainable nanotechnology involves the nanoscale control of synthesis and processing of matter without footprints that give rise to environmental degradation. Hence there is a search for synthetic methods that utilize fewer amounts of materials, water, and energy; while reducing or replacing the need for organic solvents. Notable developments include Saferby-design concepts, biologically-inert $\mathrm{SiO}_{2}$, microwave irradiation, and the use of biomass precursors $[1,2]$. Safer by design concepts in Nano synthesis has been directed towards the use of plants that contain functional compounds that facilitate the reaction [3-6]. In this regard, a multitude of natural biomaterials have been employed in the synthesis of metal nanoparticles. Examples include blackberry, blueberry, pomegranate, and turmeric extracts, the basil plant, Ocimum sanctum, and the Mangifera indica leaf $[4,7,8]$. These studies have underscored the importance of environmentally benign method as a faster synthesis compared to the chemical reduction methods.

There is a significant interest in AgNPs due to their widespread applications in catalysis, biological tagging, optics, electronics and pharmaceuticals $[3,4,7,9,10]$. Silver is historically used as antibacterial with potential to address the problem of antibiotic resistance since it is highly toxic to micro-organisms [10]. Sondi and Salopek-Sondi, for instance, reported that silver nanoparticles (AgNPs) are potent microbial agents against Escherichia coli and of all antimicrobial agents, silver elements and nanoparticle compounds have been the most extensively tested $[3,10]$.

A variety of physical, chemical, and biological techniques have been developed and used for the synthesis of AgNPs [8]. However, physical methods are costly and chemical synthesis involves 

in place of toxic chemicals and high maintenance microbes. Nadagouda and Varma conducted a study in which silver and palladium nanoparticles were synthesized using tea and coffee extracts and reported that the caffeine and polyphenols of coffee and tea form complexes with metal ions in solution while reducing them to the corresponding nanoparticles [3]. The synthesis of nanoparticles is a metal-ion reduction process. Many, if not all, of these plant extracts contain phenolic compounds such as curcuminoids and flavonoids [4, 9]. These phenols; also widely known for exhibiting antioxidant activity, are believed to be responsible for the reduction of Ag and Au cations during nanoparticle synthesis $[7,10]$.

Ghosh et al. reported the synthesis of AgNPs via the reduction of silver cations using Dioscorea bulbifera tuber extract [10]. D. bulbifera is rich in water soluble polyphenolic compounds, especially flavonoids and catechin. The results of these studies all suggested that the active species involved in biochemical synthesis of nanoparticles is phenolics, or flavonoids, however, to date no structural information was given to justify the claim [8-10]. One study, reported the 
use of quercetin to synthesize gold nanoparticles but no silver nanoparticles could be generated in aqueous solution [9].

It is worth noting that despite the broad literature demonstrating the preparation of AgNPs using phenolics $[3,4,8,7-10]$; no report has given any structural evidence or mechanistic elucidation of the exact flavonoid responsible for the reduction of $\mathrm{Ag}^{+}$to $\mathrm{Ag}^{0}$. In this paper we report for the first time the green synthesis of AgNPs using water soluble, phosphorylated and sulfonated Quercetin, including QPP, QSA, and ATRP. Prior work suggests that QCR could not reduce $\mathrm{Ag}^{+}$to $\mathrm{Ag}^{0}$ [9]. This could be attributed to its lack of solubility in water. The application of noble nanoparticles for the purification of water has received much attention and in particular the antibacterial activities of nanoparticles have been studied extensively in the recent years [13-16]. This work also shows, for the first time that indeed the active flavonoids, (QCR, QPP, QSA and ATRP) are the reducing agents leading to the formation of metal NPs which exhibit excellent antibacterial activity and cytotoxic effect. 2. Experimental Section

\subsection{Chemicals}

All chemicals were of analytical or reagent grade and were used as received from the vendors without further purification. Methylene chloride, carbon tetrachloride $\left(\mathrm{CCl}_{4}\right)$, dibenzyl phosphite, DMF, N, N-diisopropylethyllamine (DIPEA), acetonitrile, silver nitrate 99\% and methanol solvents for reaction were bought as anhydrous grade in sure seal bottles from Sigma (St. Louis, MO). Anhydrous Quercetin was purchased from MP Biomedicals, LLC, Solon, Ohio. Apigenin was purchased from Indofine Chemicals Inc. (Hillsborough, NJ). 4-dimethyl aminopyridine (DMAP), palladium 5\% on activated carbon and silver nitrate were purchased from SigmaAldrich, Milwaukee, WI. Dimethyl sulfoxide-D6 was from Cambridge Isotope Laboratories, Inc. 
MA. Methanol, hexane, ethyl acetate, sodium chloride $(\mathrm{NaCl})$, anhydrous sodium sulfate $\left(\mathrm{Na}_{2} \mathrm{SO}_{4}\right)$ and potassium dihydrogen phosphate $\left(\mathrm{KH}_{2} \mathrm{PO}_{4}\right)$ were purchased from Fisher Scientific, Pittsburg, PA. All reagents were prepared using Nanopure water with a specific resistivity of 18 $\mathrm{M} \Omega \mathrm{cm}$. All reactions involving air or moisture sensitive reagents or intermediates were performed under $\mathrm{Ar}$ or $\mathrm{N}_{2}$ atmosphere.

\subsection{Instruments}

Purification of products was carried out using Teledyne flash chromatography system. Analytical TLC was performed using $0.25 \mathrm{~mm}$ EM Silica Gel 60 F250 plates visualized by UV irradiation (254 nm). Melting point measured on Digi Melt Stanford Research Systems melting point apparatus MPA160 (USA). UV/Vis absorption spectra were obtained from an HP 8453 UVvisible diode array spectroscopy. ${ }^{1} \mathrm{H},{ }^{13} \mathrm{C},{ }^{31} \mathrm{P}$ NMR spectra were obtained using $600 \mathrm{MHz}$ Bruker Avance. TEM measurements were carried out in a JEOL TEM 2100F. XRD was performed using Philips X'pert MPD.

\subsection{Synthesis of QPP, QSA and ATRP}

The synthesis of QPP, ATRP followed the procedure described in literature [17] with slight modification in use of equivalents in chemicals for phosphorylation. However in the case of QPP and ATRP catalytic hydrogenation at ambient temperature under pressure of hydrogen was used for debenzylation purposes [18]. The synthesis of QSA followed procedure in literature [19].

\subsection{NMR - Characterization}

All $\mathrm{H}^{1}$-NMR and $\mathrm{C}-{ }^{13}$ NMR spectra were measured on a Bruker $600 \mathrm{MHz}$ spectrometer while P-NMR spectra were recorded on AC300 at $121.5 \mathrm{MHz}$ using DMSO- $\mathrm{d}_{6}$ or $\mathrm{CDCl}_{3}$ as solvent 
with $85 \% \mathrm{HPO}_{3}$ as the external reference[18]. Five aromatic protons were identified to occur between $\delta \sim 6.19 \mathrm{ppm}-\delta \sim 7.68 \mathrm{ppm} .15$ chemical shifts were identified on the $\mathrm{C}^{13}$-NMR spectrum with the carbonyl-C being assigned the chemical shift of $\delta \sim 175.85 \mathrm{ppm}$. Both $\mathrm{H}^{1}$ and C13-NMR data were consistent with the calculated theoretical chemical shifts values using ChemDraw. Table S1 is a summary of the major peaks from QCR, QPP and QSA for proton, carbon and phosphorus NMR. From these results, QPP and QSA have at least 84000 and 2700 fold enhancement in solubility over the unmodified QCR respectively.

\subsection{Synthesis of Silver Nanoparticles}

Four oven dried vial was cooled down and then purged with nitrogen. Four vials were each charged with $5 \mathrm{ml}$ of Dimethyl Formamide (DMF), $5 \mathrm{mg} \mathrm{AgNO}$ and then $2 \mathrm{mg} ; 3 \mathrm{mg}, 4 \mathrm{mg}$ and $5 \mathrm{mg}$ QCR respectively were added and then capped immediately. To optimize the conditions the experiment was set up at room temperature as follows;

1. $5 \mathrm{mg} \mathrm{AgNO}+3 \mathrm{~mL} \mathrm{DMF}+2 \mathrm{mg} \mathrm{QCR}$

2. $5 \mathrm{mg} \mathrm{AgNO}+3 \mathrm{~mL} \mathrm{DMF}+3 \mathrm{mg} \mathrm{QCR}$

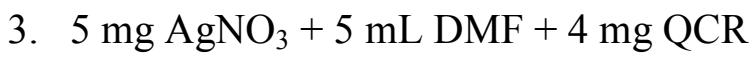

4. $5 \mathrm{mg} \mathrm{AgNO}+3 \mathrm{~mL} \mathrm{DMF}+5 \mathrm{mg} \mathrm{QCR}$

It was observed that the color change (reddish brown color) occurred immediately for QCR confirming that silver nanoparticles were formed. Quercetin is largely soluble in organic solvents and hence the use of DMF for comparison with water soluble derivatives. Quercetin is very soluble in DMF but poorly soluble in water. The Quercetin derivatives QPP, QSA and apigenin 
derivative ATRP are soluble in water and hence $5.89 \times 10^{-4} \mathrm{M}$ silver nitrate solution was prepared using water as a solvent. Four vials were each charged with $5 \mathrm{ml}$ of $5.89 \times 10^{-4} \mathrm{M}$ of $\mathrm{AgNO}_{3}$ solution then $2 \mathrm{mg}, 3 \mathrm{mg}, 3 \mathrm{mg}, 4 \mathrm{mg}$ of QPP, QCR, QSA and ATRP respectively were added separately to each vial and then capped immediately the solution was left at room temperature. The color change occurred after 8.5 hours, 1, 2 and 5 days for QCR, QPP, QSA and ATRP respectively (Figure S1). The samples for TEM were prepared by adding drops of silver nanoparticle solutions onto carbon- coated copper grids to form film layer coat on the grid and then allowed to dry before carrying out measurements on a JEOL TEM 2100F instrument.

\subsection{Antibacterial Studies}

Antibacterial activity of AgNP was tested for gram positive and gram negative bacteria. Utilizing Mueller Hinton Agar 2, different concentrations of silver nanoparticles $(20 \mu \mathrm{g} / \mathrm{mL}$ to $0.2 \mu \mathrm{g} / \mathrm{mL})$ were applied to Staphylococcus epidermidis ATCC ${ } 12228^{\mathrm{TM}}$, Escherichia coli ATCC® $25922^{\mathrm{TM}}$ and Citrobacter freundii ATCC ${ }^{\circledR} 8090$ as model microorganisms. The goal of using different concentrations of bacteria were to (i) evaluate whether the observed antimicrobial property of AgNPs depends on the concentration of bacteria inoculated onto the agar, and (ii) to check if the AgNPs show bacteriostatic and bactericidal effects depending on the nature of the AgNP and its microbial concentration.

\subsection{Cell Cytotoxicity Studies}

Cytotoxicity of AgNPs was tested at concentrations between $5-20 \mu \mathrm{g} / \mathrm{mL}$ on IEC-6 cells in DMEM medium with $0.1 \mathrm{ug} / \mathrm{mL}$ Bovine insulin, 10\% FBS and $1 \%$ penicillin \& streptomycin mixture. Cytotoxicity of Nanoparticles was tested via non-cancerous immortalized rabbit 
intestinal cell line, IEC6 cells. Different concentrations of AgNPs were tested for $10^{4}$ cells/mL for $24 \mathrm{~h}$ incubation at $37{ }^{\circ} \mathrm{C}$ under $5 \% \mathrm{CO}_{2}$ concentration.

\section{RESULTS AND DISCUSSION}

\subsection{UV- Vis characterization}

Uv/Vis Spectroscopy plays an important role in confirmation of formation of nanoparticles in aqueous medium. The formation of AgNPs was depicted by formation of reddish brown color as shown in Figure 1a and Figure S1. The formation of reddish brown color is a spectroscopic characteristic sign that AgNPs have been formed. It has been established that the color of AgNPs is due to surface plasmon resonance caused by collective oscillation of free conduction electrons majorly induced by an interacting electromagnetic field [20]. The surface plasmon of AgNPs occurred at $520 \mathrm{~nm}$ for QCR reacted with $\mathrm{AgNO}_{3}$ in DMF (Figure1a), $434 \mathrm{~nm}$ for QCR reacted with $\mathrm{AgNO}_{3}$ in deionized nanopure water (Figure 1b) and $430 \mathrm{~nm}, 404 \mathrm{~nm}$ and $399 \mathrm{~nm}$ for QPP, QSA and ATRP reacted with $\mathrm{AgNO}_{3}$ in water (Figure S2). It is worth noting the reduction of $\mathrm{Ag}^{+}$by QCR was instant in DMF while it took 8.5 hours for the $\mathrm{Ag}^{+}$to be reduced by QCR in water. The high solubility of Quercetin in DMF facilitated the faster formation of AgNPs. 
$\leadsto 2 \mathrm{mg}$ QCR

$-3 \mathrm{mg}$ QCR

$-5 \mathrm{mg} \mathrm{QCR}$

$\leftarrow 4 \mathrm{mg} \mathrm{QCR}$

Figure 1a. UV-Vis spectra of AgNPs derived from QCR. Inset is the characteristic reddish brown color depicting formation of AgNPs.

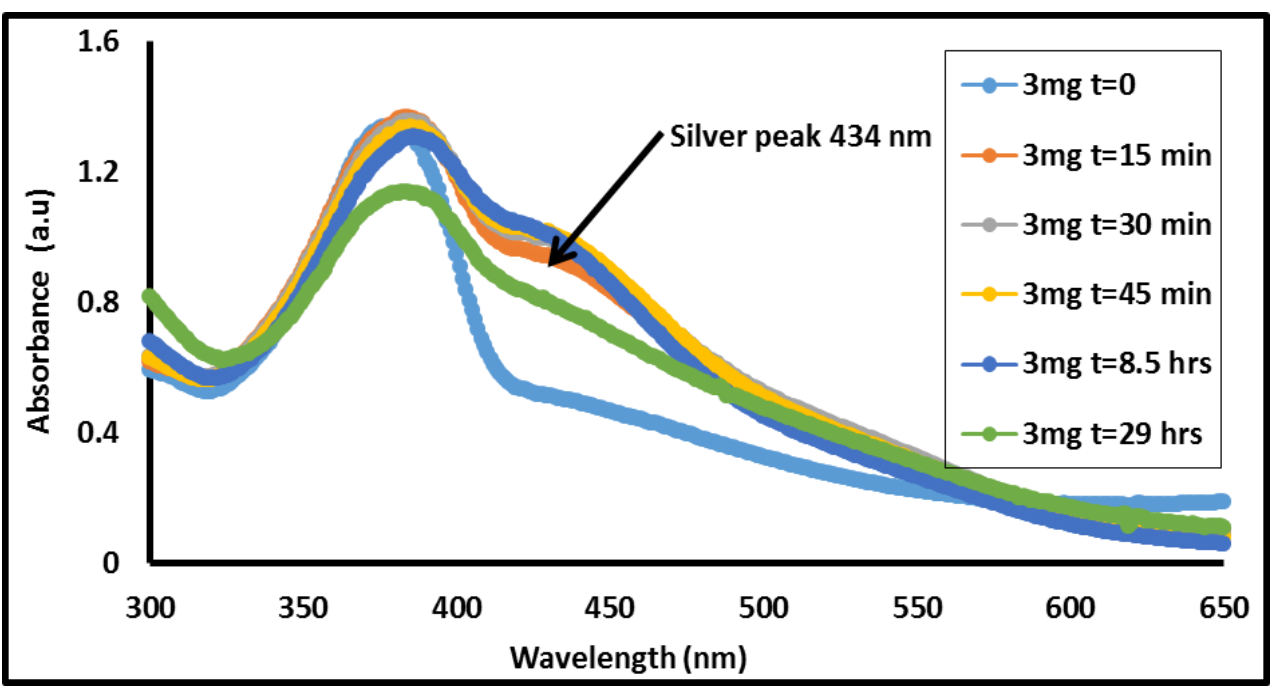

Figure 1b Uv -vis spectra of formation of AgNPs from reaction of $3 \mathrm{mg}$ of QCR $\left(9.926 \times 10^{-6}\right.$ $\mathrm{M}$ QCR) with $5.89 \times 10^{-4} \mathrm{M} \mathrm{AgNO}_{3}$ solution in deionized nanopure water from time $\mathrm{t}=0 \mathrm{~min}$ to $\mathrm{t}=29$ hours. 
Studies have illustrated that the experimentally measured surface plasmon absorption bands of silver solutions in water vary greatly in position ranging from 375 to $405 \mathrm{~nm}$ with the true position of silver in water being about $382 \mathrm{~nm}$ [20-23].

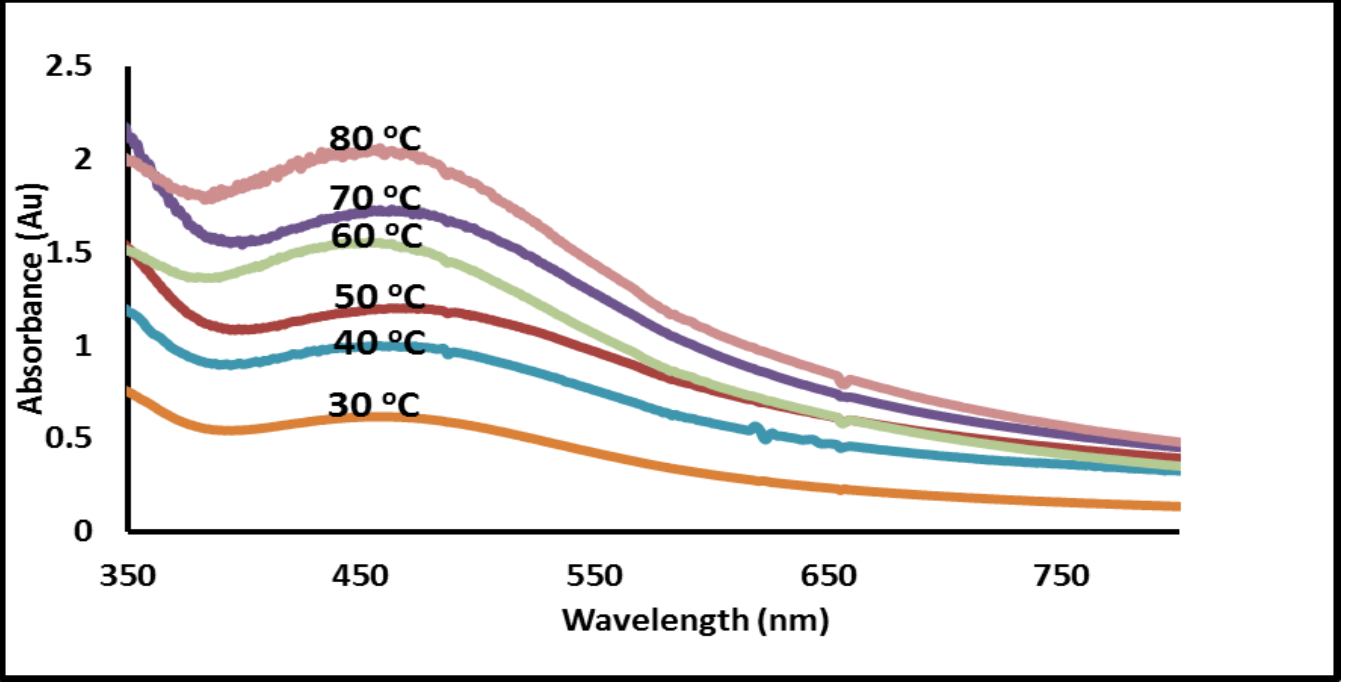

Figure 1c. UV-Vis spectra of temperature dependence on formation of AgNPs derived from QSA.

Figure 1c clearly depicts that silver nanoparticles was formed at room temperature with the highest absorbance at $80^{\circ} \mathrm{C}$. Increase in temperature led to increase in absorbance of AgNPs (Figure 1c). This clearly demonstrates that although the formation of AgNPs from these flavonoid derivatives is favored by increase in temperature, AgNPs could also be formed at room temperature $\left(25^{\circ} \mathrm{C}\right)$ and hence the synthesis of the nanoparticles could be carried out at room temperature thereby reducing the cost of production. Flavonoids are not only responsible for the reduction of $\mathrm{Ag}^{+}$to $\mathrm{Ag}^{0}$ but they also enhance efficient stabilization of nanoparticles [24]. QCR reduced $\mathrm{Ag}^{+}$instantly forming AgNPS and the characteristic brown color of AgNPs is observed. This is the first evidence of direct use of QCR for Ag nanoparticles formation from $\mathrm{AgNO}_{3}$ solution in water. Earlier reports show that AgNPs cannot be formed from aqueous solution [9]. 
After about 7 days, black precipitation occurred on the glass walls of the vial forming a lustrous silver coating (Adsorption of metallic silver on the walls).

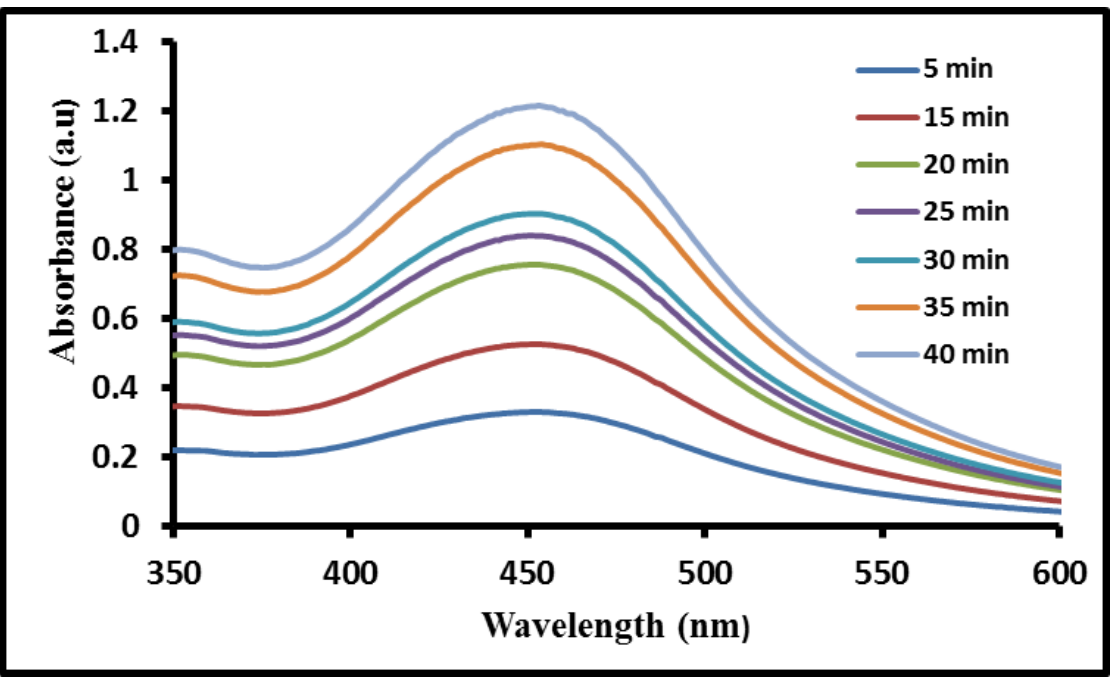

Figure 1d. Uv-vis absorption spectra of AgNPs after reaction at room temperature $\left(50^{\circ} \mathrm{C}\right)$ of $5.89 \times 10^{-4} \mathrm{M} \mathrm{AgNO}_{3}$ solution with $4.5 \times 10^{-3} \mathrm{M}$ QPP recorded at different times.

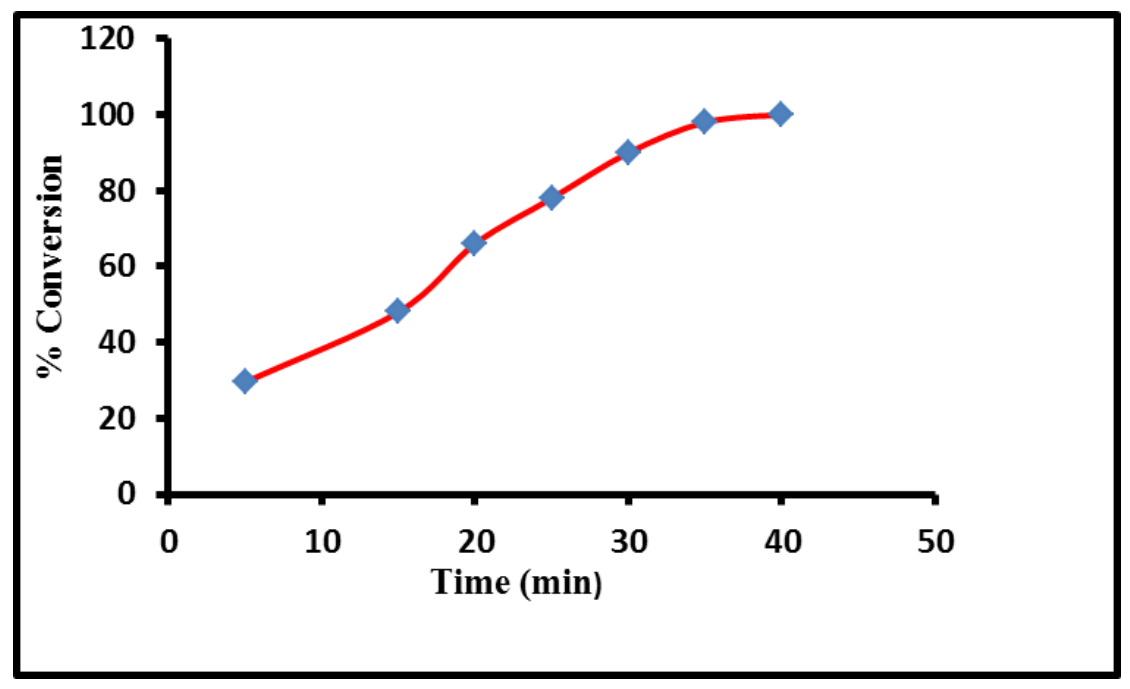

Figure 1e. Reaction progress depicting percentage conversion of AgNPs with time.

Figure 1d illustrates the formation of AgNPs with time as the reaction of QPP with $\mathrm{AgNO}_{3}$ progressed at $50^{\circ} \mathrm{C}$. After 5 minutes the silver peak occurred at $450 \mathrm{~nm}$ and the reaction reached completion after 40 minutes. The percentage conversion of $\mathrm{Ag}^{+}$to $\mathrm{Ag}^{0}$ (Figure 1e) was 
calculated based on results from Figure 1d. It is worth noting that QPP reduces $\mathrm{Ag}^{+}$to $\mathrm{Ag}^{0}$ and $90 \%$ efficiency conversion was realized after 30 minutes while $96 \%$ conversion was realized after 35 minutes.

\subsection{TEM Characterization}

The TEM images of AgNPs obtained from ATRP, QCR, QSA and QPP are shown in Figure 2 (a) to (d) respectively.
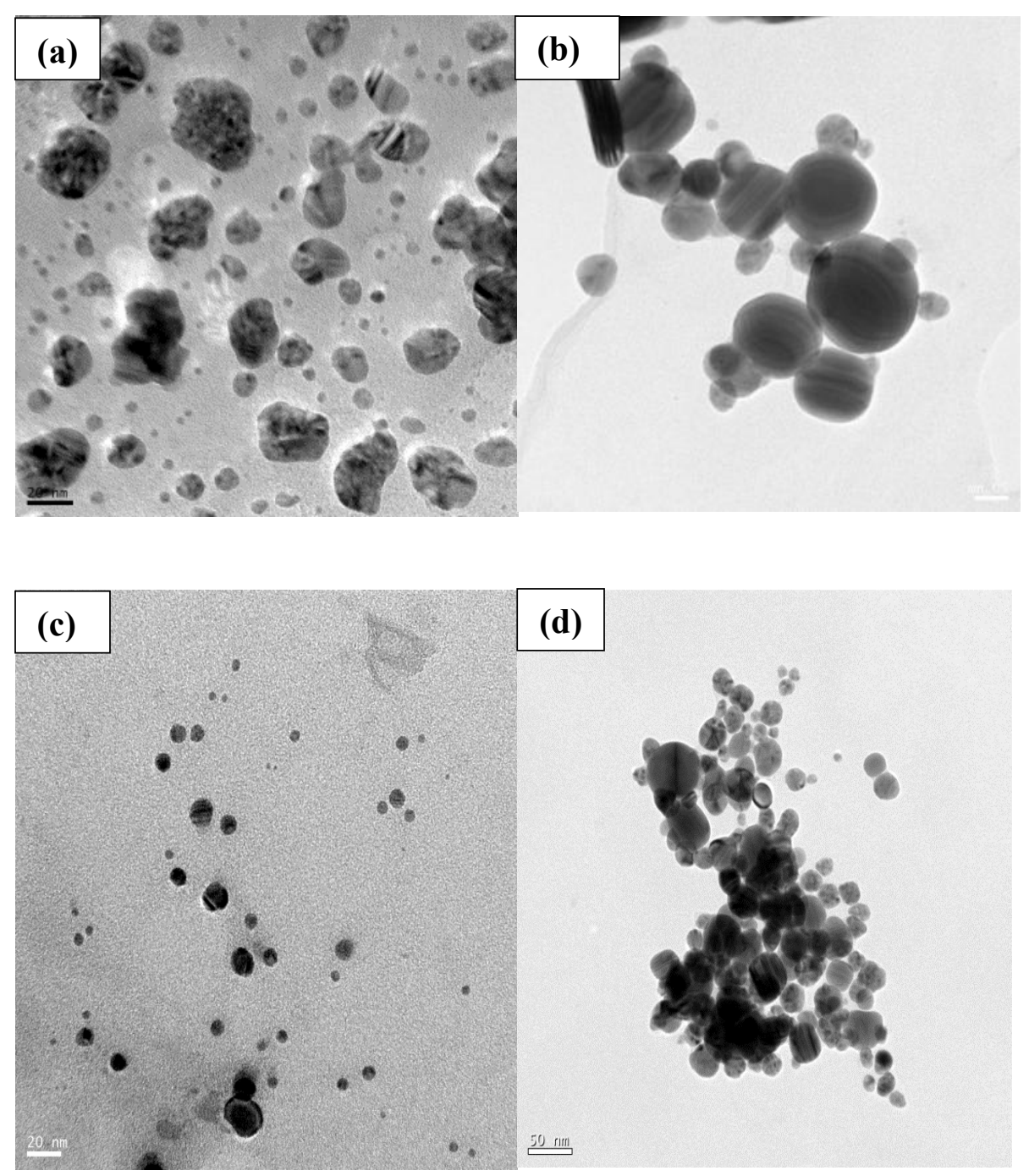

Figure 2. TEM images of AgNPs obtained using (a) QSA (b) QCR (c) ATRP and (d) QPP 
From the results, it is clearly depicted that the AgNPs obtained from QCR, QPP, QSA and ATRP were spherical in shape and the particles sizes range from 2-80 nm and 5-80 nm for QCR and QSA respectively. The nanoparticle shapes varied from spherical to hexagonal for the case of with dispersed particle size ranging from 2-30 $\mathrm{nm}$. The average size of AgNPs derived from ATRP was determined to be $10.25 \mathrm{~nm}$ (Figure S3). For QPP agglomerated particles of size range 5-50 $\mathrm{nm}$ of spherical shape with an average size of $23 \mathrm{~nm}$ (Figure S4) were formed. The high resolution TEM images (Figure-3 (a)-(d)) showed good crystalline structure of the nanoparticles. Particles were internally twinned as shown in the HRTEM images. Single crystalline particles were formed oriented in same way. Crystallinity was confirmed by the SAED spectra and the fringes observed in HRTEM images depicting that Quercetin was a good reducing agent for $\mathrm{Ag}^{+}$in water.
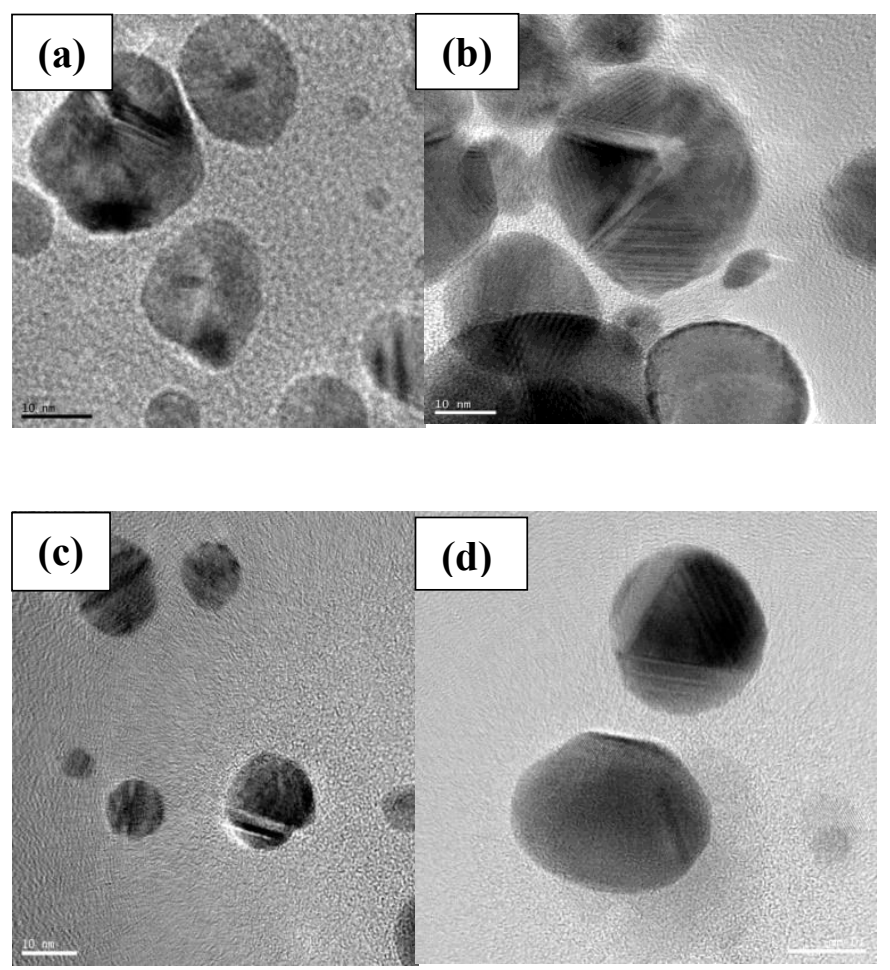

Figure 3. High resolution TEM images of AgNPs obtained using (a) QSA (b) QCR (c) ATRP and (d) QPP. 
Twinned nanoparticles with 5 fold symmetry can be seen from HRTEM (Figure-3). HRTEM images show that the nanoparticles were largely spherical in shape and also reveal internally twinned particles. The presence of well-defined lattice fringes in HRTEM demonstrates the crystalline nature of the AgNPs as depicted in Figure 3.

EDS spectra were employed to confirm formation of AgNPs as depicted in Figure 4. The EDS spectra clearly indicate the formation of $\mathrm{Ag}$ and this is demonstrated by the $\mathrm{Ag}$ peaks observed in the spectra. However, since AgNPs were drop coated on carbon coated copper grid, the peaks for $\mathrm{Cu}$ and $\mathrm{C}$ are basically from the grid used.

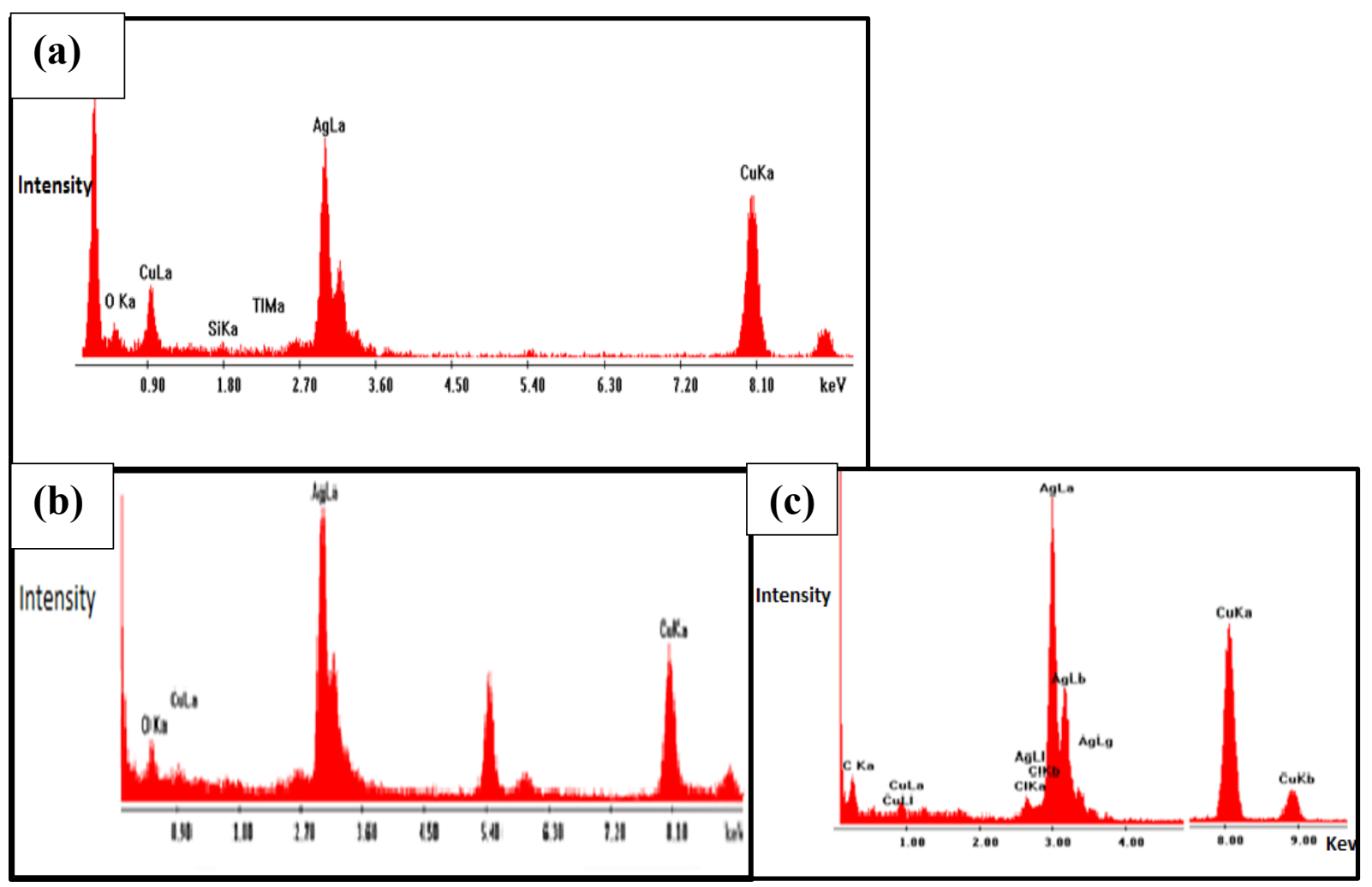

Figure 4. Energy dispersive absorption spectroscopy of AgNPs obtained from (a) QPP (b) QCR (c) ATRP.

\subsection{X-ray Diffraction Characterization}


XRD studies normally reveal the crystalline nature of sample by yielding a pattern showing numbers of Bragg's reflections which are usually indexed based on face centered cubic structure of the particle of interest. Figure 5 shows the XRD pattern of Ag nanoparticles derived from QPP. In this pattern 2 distinguished peaks were observed at $2 \theta=38.28^{\circ}, 44.43^{\circ}$ which can be indexed to correspond to (111) and (200) Braggs reflections of face centered cubic Ag nanoparticles. The diffraction peaks are in agreement with the standard value as obtained from Joint Committee on Powder Diffraction Standards (JCPDS) card No. 04-0783 [8, 25,26].

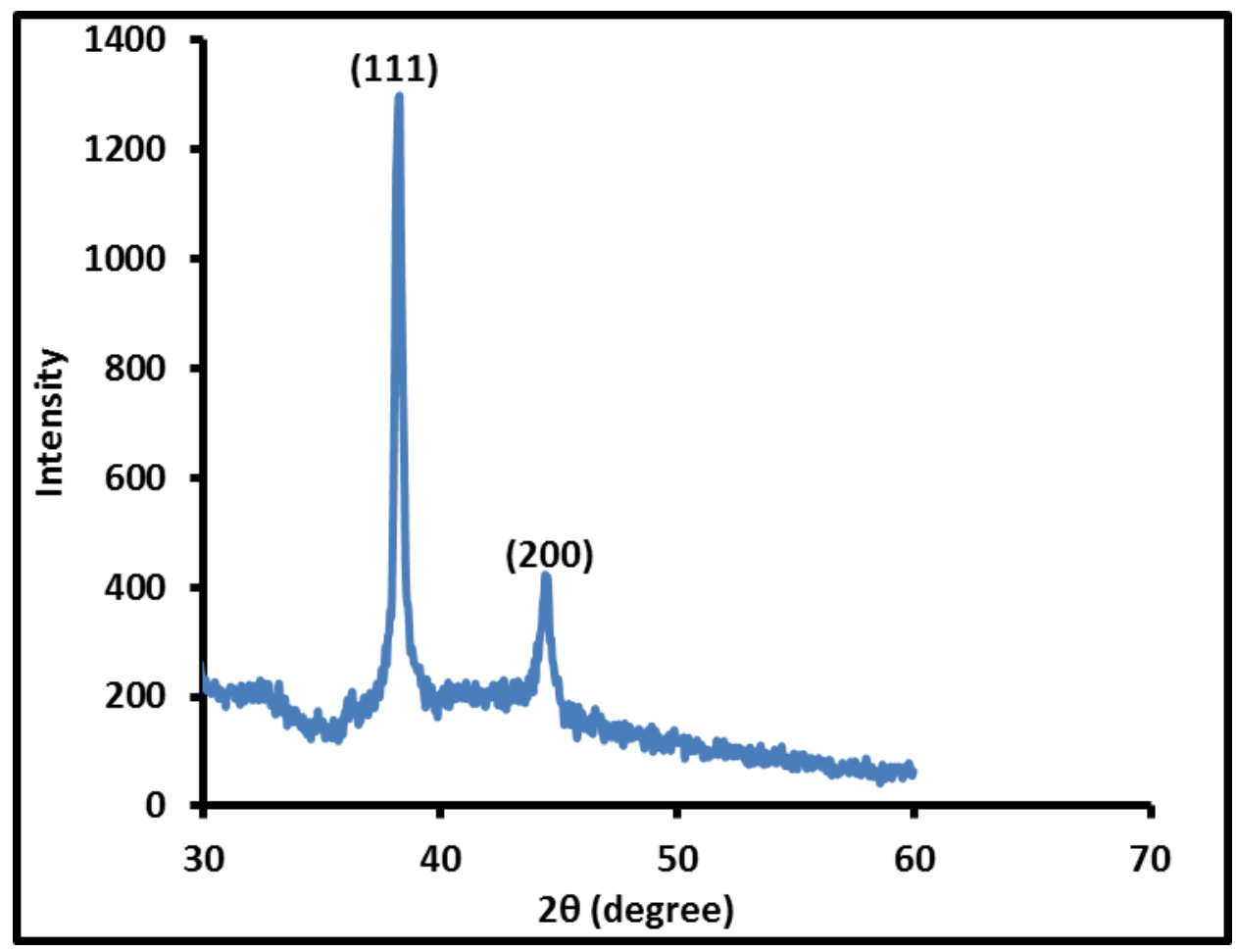

Figure 5 (a) XRD pattern of AgNPs derived from QPP

From the XRD results it is clearly evidenced that the silver nanoparticles derived from naturally derived flavonoids are crystalline in nature. Although XRD data did indicate the presence of crystal structure for some of the AgNPs, the presence of amorphous particle may still be 
possible. The flavonoid derivatives are able to reduce and stabilize the AgNPs formed. The peaks at $2 \theta=38.28^{\circ}, 44.43^{\circ}$ generally correspond to silver peaks.

The size of the Ag nano particles can be estimated by using Debye-Scherrer formula $[4,7,10,8$, $11,12] ; \mathrm{d}=\mathrm{K} \lambda / \beta \operatorname{Cos} \theta$, Where; $\mathrm{d}=$ the crystalline particle size of silver nanoparticles; $\mathrm{K}=$ the Scherrer constant (values always range from $0.9-1$ usually taken for a cubic system); $\lambda=$ the

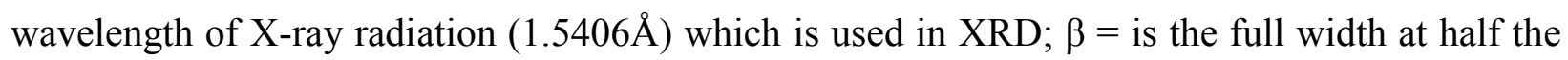
maximum intensity of the diffraction peak of (111) Brag reflection generally in radians and $\theta=$ the Bragg angle.

The formula was used to calculate the average particle size which was determined to be about 22 $\mathrm{nm}$ which is in agreement to AgNPs synthesized from past green synthesis studies from plants $[5,6]$. It is worth noting that the XRD patterns and the crystalline nanoparticle size of about 22 $\mathrm{nm}$ confirmed that our nanoparticles synthesized from naturally derived flavonoids were crystalline in nature. The results are consistent with the sizes of AgNPs obtained from TEM results (Figure S4).

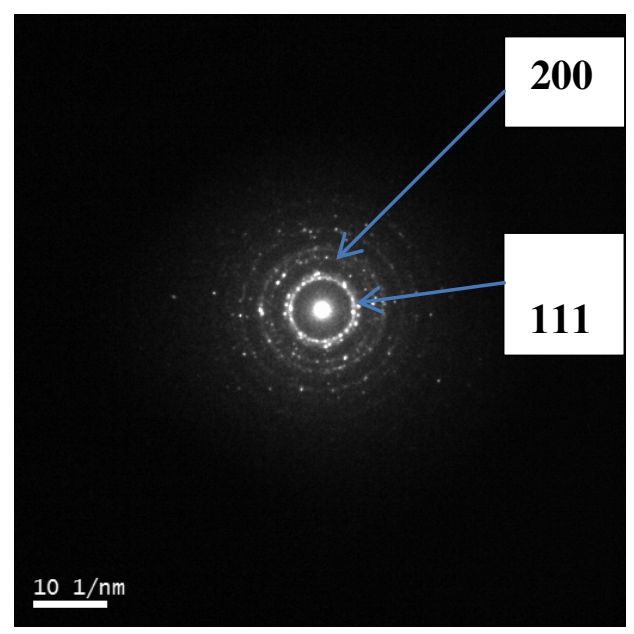

Figure 5b. Selected Area Electron Diffraction (SAED) crystallinity of AgNPs derived from QPP 
The SAED pattern confirmed the crystalline nature of the nanoparticles. This is clearly evidenced by the bright circular spots (Figure $5 \mathrm{~b}$ ) which are assigned to correspond to (111) and (200) Bragg's reflection planes. Studies based on SAED pattern revealed similar trends [8] of the pattern and hence we were able to synthesize the Ag nanocrystals obtained from naturallyderived flavonoids.

\subsection{Proposed reduction mechanism}

Quercetin is a naturally occurring flavonoid with strong antioxidant properties and is able to chelate with metal ions. Studies have revealed that naturally derived flavonoids are able to reduce chromium (VI) to chromium (III) $[18,27]$ and are able to form complexes with metals such as copper [28]. Flavonoids can also directly scavenge molecular species of active oxygen. The antioxidant action of flavonoids resides mainly in their ability to donate electrons or hydrogen atoms.
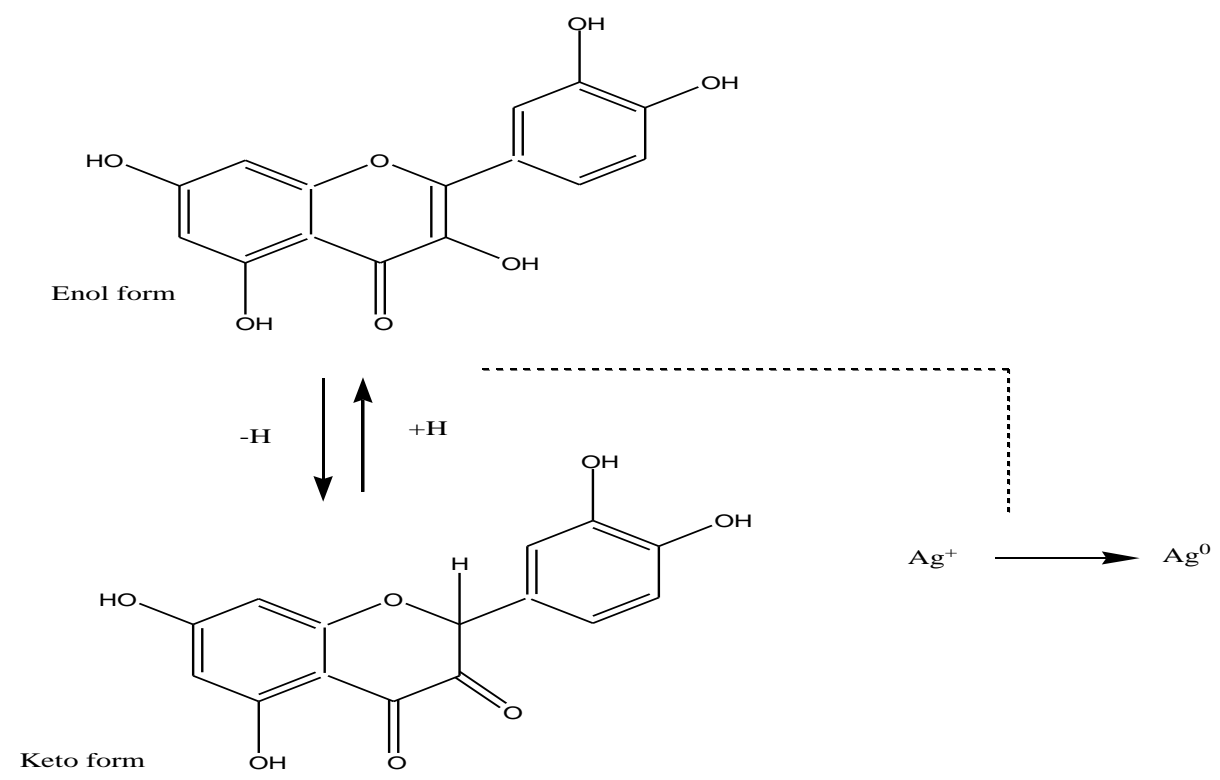

Scheme 1.Proposed mechanism of reduction of $\mathrm{Ag}^{+}$to $\mathrm{Ag}^{0}$ using quercetin 
The formation of enol form of the quercetin freely liberates reactive hydrogen which is responsible for the conversion of $\mathrm{Ag}^{+}$to $\mathrm{Ag}^{0}$ (Scheme 1). Recently a study proposed that Luteolin, a flavonoid reduces silver ions via same mechanism [7].

\subsection{Antibacterial Activity}

The antibacterial activity of AgNPs synthesized from naturally-derived macromolecules was conducted on Escherichia coli, Staphylococcus epidermidis and Citrobacter freundii. Although silver metal is a well-known antimicrobial agent, the mechanism of its bactericidal effect has been a subject of study. Some literature sources suggests that the antibacterial activity may be probably due to electrostatic attraction between negatively charged cell membrane of microorganism and positively charged nanoparticles. ${ }^{29,30}$ Silver nanoparticles possess a good chemical stability, increased surface area and small size which enhances faster interaction with microorganisms. ${ }^{31},{ }^{32}$ Furthermore, it has been reported that the particles may interact with the outer membrane and may cause degradation, structural changes leading to death ${ }^{33}$. However it is now clear that the toxicity of AgNPs may be explained via three possible mechanisms. The first mechanism may involve the release of silver ions which can interact with enzymes via thiol groups. This mechanism was established as the major pathway relevant in antibacterial activity involving $\mathrm{Ag}^{+}$ions. ${ }^{34,35 .}$ In this case, the major role of electrostatic attraction is critical. The second mechanism may involve the generation of reactive oxygen species which is fundamental in determining nanosilver toxicity. ${ }^{36}$. The third and final mechanism could be related to the penetration of nanoparticle into cell membrane leading to degradation and structural change. . $^{34,37 .}$

The study had two controls labelled (a) and (d). In this case a and d had different bacterial cultures. Control (a) was inoculated with one drop of $10^{2}$ cfu E. coli in $20 \mu \mathrm{L}$ while control (d) 
was inoculated with two drops of $10^{4}$ cfu E. coli in $20 \mu \mathrm{L}$. When the control (d) was compared with (e) and (h), it can be observed that $200 \mathrm{ng} / \mathrm{mL}$ AgNPs possessed minor inhibitory effect while $800 \mathrm{ng} / \mathrm{mL}(\mathrm{h})$ and $2 \mu \mathrm{g} / \mathrm{mL}$ AgNPs made the colonies discernible and provided strong inhibitory effect (Figure 6a).

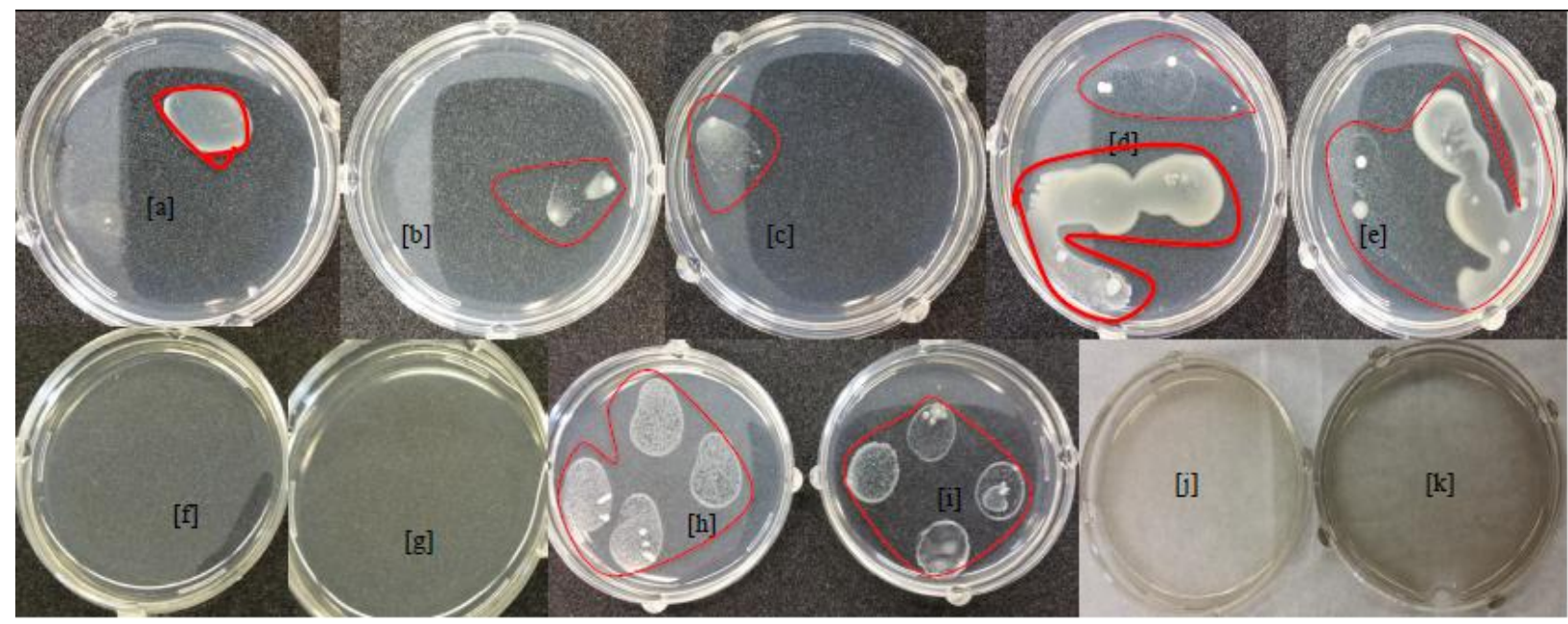

Figure 6a. Silver toxicity on E.coli ATCC® 25922 ${ }^{\mathrm{TM}}$, (a) Control, (b) $200 \mathrm{ng} / \mathrm{mL}$, (c) 800 $\mathrm{ng} / \mathrm{mL}$, (d) Control (e) $200 \mathrm{~g} / \mathrm{mL}$, and (f and h) $2 \mu \mathrm{g} / \mathrm{mL}$, (g and i) $3 \mu \mathrm{g} / \mathrm{mL}$. The control plate (a) and AgNPs treated plates (b) and (c) were incubated with one drop of $10^{2}$ and $10^{3}$ cfu E.coli in $20 \mu \mathrm{L}$. The control (d) and the AgNPs treated plates (e), (f), (g), (h) and (i) were inoculated with two drops of $10^{4}$ and $10^{5}$ cfu E.coli in $20 \mu \mathrm{L}$.

In the case of $24 \mathrm{~h}$ incubation, E.coli colonies were not visible in plates. The plates $(\mathrm{j})$ and $(\mathrm{k})$ were treated with 8 and $20 \mu \mathrm{g} / \mathrm{mL}$ AgNPs and inoculated with four drops of $10^{7} \mathrm{cfu}$ E.coli in 20 $\mu \mathrm{L}$. Despite these concentrations, no visible colony formation was seen at $72 \mathrm{~h}$ incubation. 
Figure 6b. AgNPs toxicity on C. freundii. (a) Control, (b) $200 \mathrm{ng} / \mathrm{mL}$, (c) $2 \mu \mathrm{g} / \mathrm{mL}$, (d) $3 \mu \mathrm{g} / \mathrm{mL}$, (e) $5 \mu \mathrm{g} / \mathrm{mL}$, and (f) $8 \mu \mathrm{g} / \mathrm{mL}$ AgNPs treated plates.

In all cases, $C$. freundii was introduced into the plates as two drops of $10^{4}$ and $10^{5}$ cfu in $40 \mu \mathrm{L}$ carriers. As shown in Figure 6b, the $200 \mathrm{ng} / \mathrm{mL}$ AgNPs treatment did not provide any significant toxicity on $C$. freundii growth while AgNPs started showing toxicity at $2 \mu \mathrm{g} / \mathrm{mL}$ concentration. However, 2 and $3 \mu \mathrm{g} / \mathrm{mL}$ AgNPs treatments did not inhibit the growth of $C$. freundii completely. Rather they inhibited the growth at over $99 \%$ in the case of $10^{4} \mathrm{cfu}$ inoculations while the inhibitory effect was less than $50 \%$ in the case of $10^{5} \mathrm{cfu}$ inoculations (Figure $6 \mathrm{~b}$ ). In the case of $5 \mu \mathrm{g} / \mathrm{mL}$ treatment (e), any colony formation was not seen at $72 \mathrm{~h}$ incubation. With $8 \mu \mathrm{g} / \mathrm{mL}$ AgNPs treatment (f) did not allow any bacterial colony formation at $72 \mathrm{~h}$ incubation in the case of $10^{6} \mathrm{cfu}$ inoculation in $100 \mu \mathrm{L}$. 


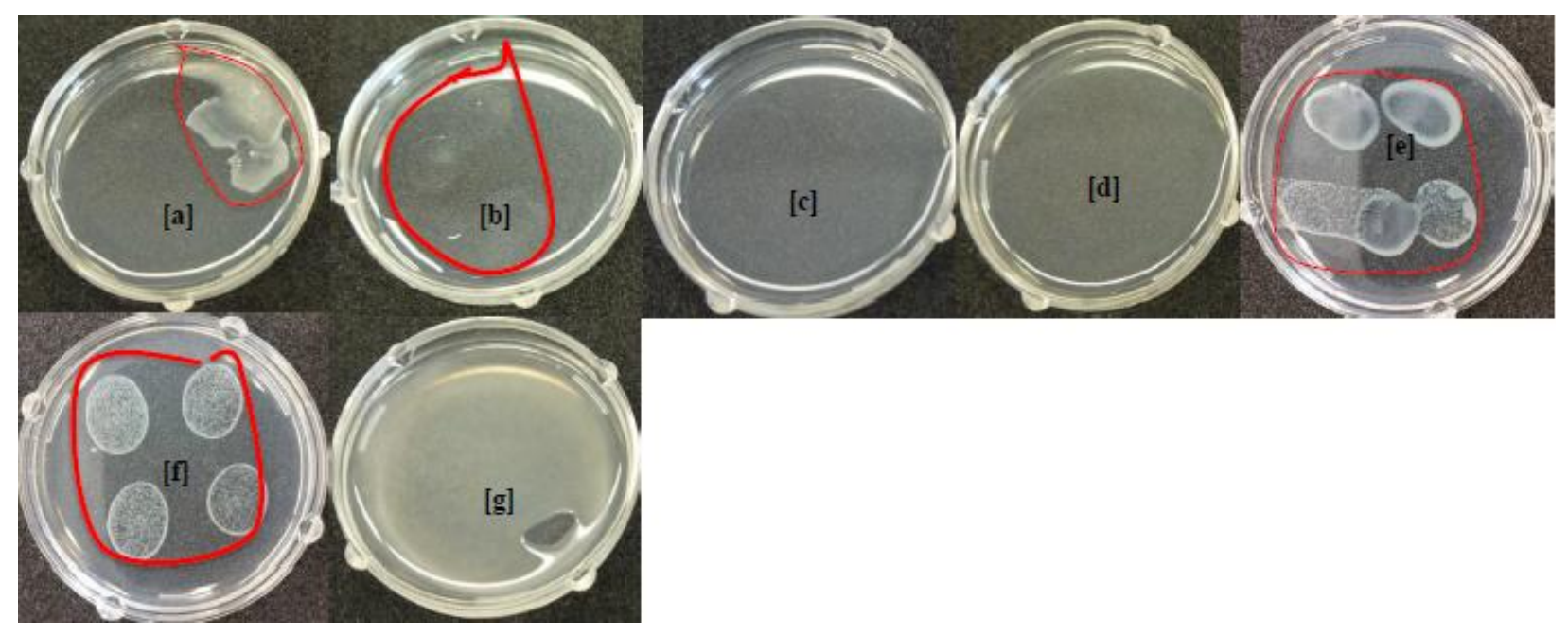

Figure 6c. Toxicity of AgNPs on S. epidermidis. (a) Control, (b) $800 \mathrm{ng} / \mathrm{mL}$ (c and e), $2 \mu \mathrm{g} / \mathrm{mL}$ (d and f), $3 \mu \mathrm{g} / \mathrm{mL}$ and (g) $5 \mu \mathrm{g} / \mathrm{mL} \mathrm{AgNPs}$ treated plates.

In all cases, S. epidermidis was introduced into the plates as two drops of $10^{4}$ and $10^{5}$ cfu in 20 $\mu \mathrm{L}$ carrier. $800 \mathrm{ng} / \mathrm{mL}$ AgNPs treatment did not possess strong significant toxicity on S.epidermidis growth at $24 \mathrm{~h}$ incubation. In the case of 2 (c) and 3 (d) $\mu \mathrm{g} / \mathrm{mL}$ AgNPs treatment, no colony formation was observed at $24 \mathrm{~h}$ while $72 \mathrm{~h}$ incubation showed abundant colony formation especially for $10^{5} \mathrm{cfu}$ inoculation (Figure $6 \mathrm{c}$ ). However, $3 \mu \mathrm{g} / \mathrm{mL} \mathrm{AgNPs}$ (f) treatment inhibited S. epidermidis growth at over $99.9 \%$ whereas at $72 \mathrm{~h}$ incubation with $5 \mu \mathrm{g} / \mathrm{mL}$ AuNPs treatment did not inhibit S. epidermidis growth at all.

AgNPs were tested for their potential bacteriostatic and bactericidal effects. This was the main reason of using different inoculum concentrations since selecting only one bacteria concentration did not give any clear idea about whether the AgNPs possess either bactericidal or bacteriostatic properties or both. The AgNPs was found to possess bacteriostatic and bactericidal effects. However, these effects were directly dependent on both NPs concentration and inoculum concentration. The age of the bacteria used in the toxicity study was kept in the log phase. 
According to the results shown in Figures $6 \mathrm{~b}$ and $6 \mathrm{c}, 5 \mu \mathrm{g} / \mathrm{mL}$ AgNPs exhibited significant growth inhibition at $10^{5} \mathrm{cfu}$ inoculum. Even though the concentrations below $5 \mu \mathrm{g} / \mathrm{mL}$ AgNPs showed some bactericidal effects, their effect on the bacteria were more bacteriostatic in nature. Based on these results, $5 \mu \mathrm{g} / \mathrm{mL}$ AgNPs can be accepted as a strong bactericidal agent to eliminate the tested bacteria at up to $10^{5} \mathrm{cfu}$ inoculum. In the case of $8 \mu \mathrm{g} / \mathrm{mL}$, the concentration can be increased to $10^{6}$ and $10^{7}$ cfu inoculum. Altering the addition of AgNPs to the agar did not provide any significant difference.

The antibacterial activity of the synthesized AgNPs was compared with $\mathrm{AgNO}_{3}$ salt by dissolving the $\mathrm{AgNO}_{3}$ solution in Mueller Hinton Agar at different concentrations. Results showed that $\mathrm{AgNO}_{3}$ salt solution at $1 \mu \mathrm{g} / \mathrm{mL}$ concentration was enough to wipe-out each of 100 $\mu \mathrm{L}$ of $10^{7} \mathrm{cfu} / \mathrm{mL}$ E.coli, C. freundii and S. epidermidis microbial inoculums [Figure S5]. This is expected because silver ions have better natural interaction with microbes. As discussed in the Supplementary Section, adding AgNPs to the agar before autoclaving slightly increased the antibacterial activity of the nanoparticles. The particles were also tested for cytotoxic activities as depicted in the supplementary section (Figure S6 and S7).

\subsection{Conclusion}

We have successfully synthesized greener AgNPs for the first time using flavonoid derivatives QPP, QSA and ATRP using water as the solvent and without using any capping agents. UV-vis spectrometry was used to confirm the synthesis of AgNPs based on the presence of plasma resonance peak while TEM and HRTEM were employed to determine the shape and size of silver nanoparticles. The particle size ranged from 2- $80 \mathrm{~nm}$ depending on the flavonoid used. XRD results confirmed that the Ag nanoparticles obtained from naturally- derived flavonoids 
were crystalline in nature with average nanoparticle size of $22 \mathrm{~nm}$. The greener synthetic approach is also an indication that it could be used at industrial scale as a synthetic protocol. The synthesized AgNPs exhibited excellent antibacterial activity against E.coli, S. epidermidis and C. freundii.

\section{Acknowledgements}

The authors acknowledge the National Science Foundation CBET 1230189 for funding. The Regional NMR facility (600 MHz instrument) at SUNY-Binghamton is supported by NSF (CHE-0922815).

\section{Appendix A: Supporting data.}

Additional information showing major NMR chemical shifts and solubility of QCR, QSA and QPP derivatives; color changes depicting the formation of silver nanoparticles; UV/Vis spectra of silver nanoparticles peaks derived from QPP, QSA and ATRP and histogram showing the average particle size. The cytotoxicity studies results are also available. These materials are available free of charge via Internet at http://dx.doi.org/............

\section{References}

1. Sadik O. A., Du N., Kariuki V., Okello V., \& Bushlyar V.,2014. Current and Emerging Technologies for the Characterization of Nanomaterials, ACS Sustainable Chemistry \& Engineering, 2 1707-1716.

2. Sadik O. A., Yazgan I., Kariuki V., 2014. Sustainable nanomaterials derived from synergistic properties of nanomaterials and biomacromolecules, Chemical Processes for a Sustainable Future, eds. T. M. Letcher, J. L. Scott and D. Patterson, Royal Society of Chemistry, Cambridge, UK, 27 chapters, .... Pages, ISBN: 978-1849739757,

3. Nadagouda, M.N and Varma, R.S., 2008. Green synthesis of silver and palladium nanoparticles at room temperature using coffee and tea extract. Green Chemistry 10 859-862. 
4. Nadagouda, M.N.; Iyanna, N.; Lalley, J.; Han, C.; Dionysiou, D. D.; Varma, R.S., 2014. Synthesis of Silver and Gold Nanoparticles Using Antioxidants from Blackberry, Blueberry, Pomegranate, and Turmeric Extracts. ACS sustainable. Chem. Eng. 2 1717-1723.

5. Awwad, A.M. and Sale m, N.M., 2012. Green Synthesis of Silver Nanoparticles by Mulberry Leaves Extract. Nanoscience and Nanotechnology 2 (4) 125-128.

6. Kouvaris, P.; Delimittis, A.; Zaspalis, V.; Papadopoulos D.; Tsipas, S.A.; Michailidis, N.,2012 Green synthesis and characterization of silver nanoparticles produced using Arbutus unedo leaf extract. Materials Letters 76 18-20.

7. Ahmad, N.; Sharma, S.; Alam, K .Md.; Singh, V.N.; Shamsi, S.F.; Mehta, B.R.; Fatma, A, 2010 Rapid synthesis of silver nanoparticles using dried medicinal plant of basil. Colloids and Surfaces B: Biointerfaces 81: 81-86.

8. Philip, D.,2011. Mangifera Indica leaf-assisted biosynthesis of well-dispersed silver nanoparticles. Spectrochimica Acta Part A 78: 327-331.

9. Begum, N.A.; Mondal, S.; Basu, S; Laskar, R.A.; Mandal, D., 2009 Biogenic synthesis of $\mathrm{Au}$ and $\mathrm{Ag}$ nanoparticles using aqueous solutions of Black Tea leaf extract. Colloids and Surfaces B: Biointerfaces 71) 113-118.

10. Ghosh, S.; Patil, S.; Ahire, M.; Kitture, R.; Kale, S.; Pardesi, K.;Cameotra, S.S.; Bellare, J.; Dhavale, D.D,; Jabgunde, A.; Chopade, B. A.,2012. Synthesis of silver nanoparticles using Dioscorea bulbifera tuber extract and evaluation of its synergistic potential in combination with antimicrobrial agents. International Journal of Nanomedicine.7, 483-496.

11. Christy, A.J. and Umadevi, M.,2012. Synthesis and characterization of monodispersed silver nanoparticles. Adv. Natsci. Nanotechnol.3: 035013.

12. Agnihotri, S.; Mukherji, S.; Mukherji, S, 2013. Immobilized silver nanoparticles enhance contact killing and show highest efficacy: elucidation of the mechanism of bactericidal action of silver. Nanoscale 5, 7328-7340.

13. Bindhu, M.R.;Umadevi,M, 2015. Antibacterial and catalytic activities of green synthesized silver nanoparticles, Spectrochimica Acta Part A: Molecular and Biomolecular Spectroscopy,135, 373-378.

14. Rathi Sre, P.R.; Reka,M.; Poovazhagi,R.; Kumar,A.;Murugesan,K.,2015. Antibacterial and cytotoxic effect of biologically synthesized silver nanoparticles using aqueous root extract of Erythrina indica lam, Spectrochimica Acta Part A: Molecular and Biomolecular Spectroscopy, 135, 1137-1144.

15. Singh, K.;Pangal,M.;Kadyan,S.; Chaudhary,U.;Yadav, J.P.,2014. Antibacterial Activity of Synthesized Silver Nanoparticles from Tinospora Cordifolia against Multi Drug resistant Strains of Pseudomonas aeruginosa Isolated from Burn Patients,J. Nanmed Nanotechnol, 5:2.

16. Bindhu, M. R.; Rekha, P. V.; Umamaheswari, T.; Umadevi, M.,2014. Antibacterial activities of Hibiscus cannabinus stem-assisted silver and gold nanoparticles, Materials Letters, , 131, 194-197.

17. Silverberg L. J.; Dillon J. L.; Vemishetti P., 1996. Simple, Rapid and Efficient protocol for the selective phosphorylation of phenols with Dibenzyl Phosphite. Tetrahedron Lett. 37(6): 771 - 774. 
18. Okello, V.A.; Zhou,A.; Chong,J.; Knipfing, M.T.; Doetschman, D \& Sadik, O.A.,2012. Reduction of Hexavalent Chromium using Naturally-derived Flavonoids, Environmental Science \& Technology, 46 (19) 10743-10751.

19. Kopacz, M.,2003. Quercetin and morinsulfonates as analytical reagents. J. Anal. Chem. 58 (3), 225-229.

20. Mulvaney, P.,1996. Surface Plasmon Spectroscopy of Nanosized Metal Particles. Langmuir, 12, 788-800

21. Miller, W.J. and Herz, A.H.,1976. Characterization and Oxidation of Colloidal Silver. Colloid and Interface Science, vol. IV. Hydrosols and Rheology, M. Kerner, (ed.).Academic Press. pp. 315-329.

22. Heard, S. M.; Grieser, F.; Barraclough, C. G.; Sanders, J. V.,1983. J. Colloid Interface Sci., 93, 545.

23. Linnert, T.; Mulvaney, P.; Henglein, A.,1993. J. Phys. Chem., 97,679.

24. Ling, L.T.; Yap, S.A.; Radhakrishnan, A.K.; Subrahmanian, T., Cheng, H.M.; Palanisamy, U.D., 2009. Standardized Mangifera indica extract is an ideal antioxidant. Food Chem., 113 (4).

25. Xu, G.; Qiao, X.; Qiu, J.; Chen, J., 2008. Colloid Surf. A), 320, 222-226.

26. Wang, H.; Qiao, X.; Chen, J.; Ding, S.,2005 Colloid Surf. A 256, 111-115.

27. Sadik, O.A.; Okello, V.; Osonga, F.; Omole,M.A.; K'Owino, I.,2014. Green remediation of hexavalent chromium using naturally-derived flavonoids and engineered nanoparticles, Nanotechnology Applications Vol. 2: Solutions for Improving Water Quality, ElsevierChapter 29 pg 457-470.

28. Bukhari,S.B.;Memon,S.;Mahroof-Tahir,M.;Bhanger,M.I.;

2009. Synthesis, characterization and antioxidant activity copper-quercetin complex. Spectrochim Acta Part A71, 1901-1906.

29. Bindhu, M.R.; Umadevi, M., 2014. Antibacterial activities of green synthesized gold nanoparticles. Materials Letters, 120, 122-125

30. Sondi, I.; Salopek-sondi, B., 2004. Silver nanoparticles as antimicrobial agent: a case study on E. coli as a model for Gram-negative bacteria. J. Colloid Interface Sci. 275, 177-182.

31. Sreekanth, T.V.M.; Nagajyothi, P.C.; Supraja, N.; Prasad, T.N.V.K.V.,2015. Evaluation of the antimicrobial activity and cytotoxicity of phytogenic gold nanoparticles, Appl. Nanoscience, 5,595-602

32. Nirmala, G.A.; Pandian, K., 2007 Antibacterial efficacy of aminoglycosidic antibiotics protected gold nanoparticles- a brief study. Collo. Surfa A. Physicochem Eng. Aspects, 297:63

33. Zawrah, M.F.; Sherein, I.,2011. Antibacterial activities of gold nanoparticles against major foodborne pathogens, Life Sci J, , 8:37

34. Liu,J.; SonShine, D.A.; Shervani,S.; Hurt, R.H.,2010. Controlled Release of Biologically Active Silver from Nanosilver Surfaces. ACS Nano, 4 (11), 6903-6913.

35. Liu,J.; Hurt,R.H.,2010. Ion release Kinetics and Particle Persistence in Aqueous Nano-Silver Colloids. Environmental Science \& Technology 44, (6), 2169-2175.

36. Xia, T.; Kovochich, M.; Monty, L.; Madler, L.; Gilbert ,B.; Shi, H.; Yeh, J.I.; Zink, J.I.; Nel, A.E.,2008. Comparison of the Mechanism of Toxicity of Zinc Oxide and Cerium Oxide Nanoparticles Based on Dissolution and Oxidative Stress Properties. ACS Nano 2, (10), 2121-2134. 
37. Choi, O.; Hu, Z., 2008. Size dependent and reactive oxygen species related to nanosilver toxicity to nitrifying bacteria. Environmental Science and Technology $42,(12), 4583-4588$. 
